\title{
Estudio de caso de la implementación de la evaluación de las competencias de los jugadores de fútbol en la categoría cadete del Sevilla F.C. Case study of the implementation of a competence evaluation of soccer players in the «cadete» category of Sevilla F.C.
}

\author{
*Carlos Bryant Lazaro Paulina, *Ana María Gallardo Guerrero, **Marta García Tascón \\ *Universidad Católica San Antonio de Murcia (España), **Universidad Pablo de Olavide (España)
}

Resumen. El fútbol es uno de los deportes más practicados del mundo, exigiendo a entrenadores, técnicos de clubes y asociaciones especializadas en el deporte disponer de herramientas para identificar potenciales jugadores que demuestren un alto nivel profesional. Es por ello que el objetivo fue implementar una metodología de evaluación para el análisis de un modelo competencial del jugador de fútbol (MCJF) en dos equipos de la categoría cadete del Sevilla FC. Se analizó la percepción competencial de 4 dimensiones (psicológicas, tácticas, técnicas y físicas), en una escala de seis niveles. Se realizó un análisis bidireccional, donde se compara la percepción del jugador sobre sí mismo y la percepción del entrenador sobre el jugador, y si ésta depende del puesto ocupado en el campo. Los resultados sugirieron que la valoración sobre la percepción de las competencias para detectar el talento de entrenadores y jugadores son similares para todas las dimensiones, destacando la táctica, técnica y psicosocial. La percepción que tiene un jugador sobre la valoración de sus competencias depende del puesto que ocupa en el terreno de juego. En cuanto a la percepción del entrenador, las dimensiones táctica y técnica mostraron dependencia de la variable analizada. Este estudio muestra la necesidad de disponer de herramientas no sólo para los entrenadores en la detección del talento del deportista, sino también para que los propios jugadores conozcan las expectativas que se espera de ellos para desarrollar un juego de alto nivel profesional.

Palabras clave: Futbolista, cadete, valoración, dimensiones, competencias.

\begin{abstract}
Soccer is one of the most practiced sports in the world, requiring coaches, club technicians and specialized sports associations to have the tools to identify potential players who demonstrate a high professional level. That is why the objective was to implement an evaluation methodology for the analysis of a soccer player competency model (MCJF) in two teams in the Sevilla FC «cadete» (U15-16) category. The competence perception of 4 dimensions (psychological, tactical, technical and physical) was analyzed, on a scale of six levels. A bidirectional analysis was carried out, where the player's perception of himself and the coach's perception of the player are compared, and if this depends on the position held on the field.

The results suggested that the assessment of the perception of skills to highlight the talent of coaches and players are similar in all areas, highlighting the tactical, technical and psychosocial dimensions. The perception that a player has about the assessment of his skills depends on the position he occupies on the field of play (ANOVA $p<0.05$ ). Regarding the perception of the coach, the tactical and technical dimensions showed dependence on the variables studied (ANOVA p <0.05). This study shows the need for tools not only for coaches to detect talent, but also for the players themselves to know what's expected of them to develop at a professional level.
\end{abstract}

Keywords: Footballer, cadete, assessment, dimensions, competencies.

\section{Introducción}

El fútbol es una de las disciplinas deportivas más practicadas e influyentes a nivel mundial (Fédération Internationale de Football Association, FIFA, 2009; Ceballos-Gurrola et al., 2020). Aproximadamente 6.7 millones de aficionados en todo el mundo y 2.600 millones de personas participando directamente en el fút-

Fecha recepción: 28-07-21. Fecha de aceptación: 27-08-21

Carlos Bryant Lazaro Paulina

carlos.bryant.lazaro@gmail.com bol (Peña et al., 2017). Por ello, este deporte es objeto de estudio, no sólo desde el punto de vista deportivo, sino desde un enfoque multidisciplinar (Devís-Devís et al., 2010).

Una de las principales necesidades de los clubes y asociaciones especializadas en el fútbol, es conseguir el mayor número de jugadores que demuestren un alto rendimiento deportivo (Carmichael et al., 2001; González-Víllora et al., 2011). González-Campos et al. (2015) señalan que para analizar con precisión el rendimiento de un jugador es necesario realizar una exploración multifactorial de todos los factores influyentes, es 
decir, realizar una evaluación multidimensional. Las dimensiones que engloban la evaluación del rendimiento deportivo se enfocan en cuatro grandes categorías que influyen directamente en el jugador de fútbol. Se destaca los aspectos técnicos y tácticas, la condición física y los aspectos psicológicos. En estos últimos consideran todos aquellos factores del entorno que repercuten directamente en el deportista (Ceballos-Gurrola et al., 2020; González-Campos et al., 2015; Martínez-Cabrera et al., 2020; Vera-Haro et al., 2015).

No obstante, otro aspecto a considerar en el fútbol es su imprevisibilidad, dificultando la evaluación de los jugadores (Monteleone \& Ortega, 2015; Reilly et al., 2000a) y por tanto la selección de los instrumentos más adecuados para ello. De ahí que los criterios para la selección de los jugadores difieren entre los países y clubes, donde cada uno ha establecido sus propios programas, los cuales la mayoría no están desarrollados de forma sistemática o estructurada, si no que utilizan datos observacionales obtenidos a partir de pruebas físicas y técnicas (Fernandes et al., 2019; Reilly et al., 2000b).

Numerosas investigaciones han utilizado instrumentos con el fin de recoger datos que permitan la evaluación de las dimensiones físicas, psicológicas, técnicas y tácticas en el ámbito de las ciencias de la actividad física y el deporte (De Mata, 1999; Ortega et al., 2008; González-Ponce et al., 2018; Vidarte et al., 2019). Sin embargo, como indica Prieto (2018) muchos de ellos carecen de método científico y están creados de forma analítica sin tomar en cuenta aspectos relevantes como la autoevaluación y la perspectiva del entrenador y jugador.

Otros autores señalan que en el fútbol de categorías inferiores la intuición junto con la percepción de los entrenadores, desarrollada por medio de la observación de los jugadores, es la forma de evaluación más adecuada y que debería de tener una mayor importancia en el rendimiento deportivo y selección de los jóvenes talentosos (Barreto et al., 2020; Cano, 2001; Krogh, 2009; Holt \& Mitchell, 2006; Prieto, 2020). De forma general, en el deporte siempre se está en la búsqueda de conocer las percepciones de los entrenadores hacia los jugadores e implementar programas que garanticen el buen rendimiento de los deportistas (Urbano et al., 2020; Nuviala-Nuviala et al., 2021). A pesar de esto, son pocos los instrumentos de evaluación validados que permiten la evaluación de las dimensiones física, psicológica, técnica y táctica por medio de la percepción.

Autores como Abad et al. (2013) y Prieto-Andreu (2020) han manifestado que la percepción que ofrecen los entrenadores es fundamental durante las valoraciones de las competencias evaluadas y durante la formación del jugador, siendo, por lo tanto, un elemento importante en el fútbol. Además de esto, el rol que ejerce la opinión del entrenador puede determinar el éxito en los jóvenes futbolistas (Ballester et al., 2020; Mendo \& Morales, 2010; Paixao et al., 2019; Pulido et al., 2016; Urbano et al., 2020). Esta forma de evaluación se encuentra fundamentada por los conocimientos adquiridos por el entrenador o experto (Ballester et al., 2020; Fernández-Rio \& Méndez-Giménez, 2014; Pazo-Haro et al., 2011; 2012).

Una de las particularidades de mayor importancia de la valoración de jugadores desde la percepción es que la evaluación se realiza en base a observaciones repetidas, permitiendo captar lo que acontece en la realidad y tener en cuenta todas las competencias y cómo el jugador puede relacionarlas. La intuición o percepción no están determinadas, sino viene dada por años de entrenamiento, experiencia y aprendizaje del entrenador (Ballester et al., 2020; López-Ros et al., 2014; Santos et al, 2010; Urbano et al., 2020). De esto, Martín-Barrero et al. (2020) pretendieron establecer los patrones de juego de un equipo profesional de Alemania mediante la observación de partidos y mediante la perspectiva de varios entrenadores. Esta metodología permitió valorar el equipo desde un punto objetivo. Un estudio similar fue realizado por Caicedo-Parada \& CalderónVargas (2020) al utilizar un instrumento observacional para la valoración de acciones tácticas ofensivas en fútbol. En este estudio la observación y percepción fueron los factores claves de evaluación.

De igual forma, una característica propia de esta forma de evaluación viene dada en que las percepciones de los entrenadores pueden afectar el rendimiento deportivo de los jugadores (Ballester et al., 2020; GonzálezCampos et al., 2015; Martínez-Cabrera et al., 2020; Urbano et al., 2020). Un estudio realizado por GarcíaMas et al. (2011) destaca que los jugadores de fútbol base perciben claramente sus competencias deportivas, las cuales se vieron afectadas por la percepción del entrenador.

En este sentido, se puede resaltar un efecto asociado a la valoración desde la perspectiva del entrenador. Hancock et al. (2013) señalaron que existen fenómenos que se establecen en la interacción entre el entrenador y los jugadores. Uno de ellos es el efecto Pigmalión, el cual se basa en la existencia de una mayor expectativa de los entrenadores hacia el deportista más hábil. Esto puede afectar de manera positiva su rendimiento de- 
portivo, resultado en que ese atleta reciba más instrucción y mayor atención en comparación a otros jugadores (Casamichana \& Castellano, 2009; Castillo, 2014; Hancock et al., 2013). Por lo tanto, la valoración de los jugadores por medio de la percepción del entrenador puede verse afectada por el efecto Pigmalión (Krahenbühl \& Leonardo, 2020), siendo este hecho visto ampliamente en el fútbol, desde categorías base a categorías más importantes.

Por otra parte, la evaluación multifactorial de los factores físicos, psicológicos, técnicos y tácticos por medio de la percepción puede ser realizada por los mismos jugadores de fútbol, lo cual permitiría aumentar sus conocimientos en cuanto a sus destrezas, habilidades y funcionamiento como equipo (Adanis et al., 2017; Tapia-Flores \& Hernández-Mendo, 2011). Prieto-Andreu et al. (2020) afirman que la autoevaluación en el fútbol es un elemento clave, ya que «autoevaluarse» es la capacidad del jugador para juzgar sus competencias y habilidades respecto a los entrenamientos o partidos, permite descubrir cómo fueron logradas determinadas tácticas de juego, permite situar al jugador con respecto a los demás compañeros del equipo y reconocer que se debe mejorar (Lago-Ballesteros et al., 2012).

Las percepciones crean las bases para las actitudes y las posibles actuaciones de los jugadores, considerando también que modificando sus actitudes se pueden llegar a modificar sus acciones y la toma de decisiones (Almonacid-Fierro et al., 2020). De este modo, no sólo importa las valoraciones de los entrenadores, sino también cómo perciben los jugadores sus competencias deportivas y las condiciones en que el deporte se desarrolla (Prieto-Andreu et al., 2020). Por esta razón, es necesario conocer la percepción de los jugadores.

Es de destacar que en la autoevaluación de los jugadores el efecto Galatea juega un papel muy importante, ya que este efecto se basa en que las expectativas puestas en algunos jugadores les brindan a dichos deportistas una mejor percepción de ellos mismos, aumentando su percepción en cuanto a desempeño y autoeficiencia (Hancock et al., 2013). Tales señalan que la percepción del entrenador interfiere directamente con la oportunidad de participación y desarrollo deportivo del deportista (Krahenbühl \& Leonardo, 2020).

En vista de lo descrito, se hace patente que, tratándose de una competición deportiva tan importante, es necesario contar con herramientas adecuadas que permitan la valoración y seguimiento de cada una de las dimensiones involucradas en el rendimiento deportivo por medio de la percepción. En este sentido, Lázaro et al. (2019), han diseñado un instrumento válido y fiable para la recolección de datos a través de un cuestionario sistemático donde evalúan 4 dimensiones (física, psicológica, técnica y táctica) para determinar el nivel de competencia y rendimiento deportivo en jugadores de fútbol en España, especialmente diseñado para las categorías infantil, cadete y juvenil. Este Modelo Competencial del Jugador de Fútbol (MCJF) es un instrumento que recaba información de la percepción tanto de los entrenadores como de los jugadores de las citadas dimensiones, analizando de forma precisa la capacidad que el jugador tiene para resolver diferentes situaciones durante un partido.

Por ello, el objetivo principal de este presente trabajo es aplicar el MCJF y analizar la percepción que tiene el entrenador sobre la valoración de las competencias psicológicas, tácticas, técnicas y físicas de los jugadores de la categoría cadete del Sevilla F.C. Como objetivos secundarios se marcan:

a) analizar la comparativa del entrenador respecto a la autopercepción que tiene el jugador de fútbol aplicando el MCJF

b) analizar el objetivo principal en función del puesto que ocupa el jugador en el terreno de juego

Como hipótesis de trabajo se plantean; H1: Existen diferencias significativas entre las percepciones del jugador y del entrenador en las diferentes dimensiones (psicológica, física, técnica y táctica); H2: Existen diferencias significativas entre las percepciones del jugador y del entrenador en las diferentes dimensiones (psicológica, física técnica y táctica) respecto a la posición del jugador en el campo.

\section{Material y método}

\section{Participantes}

La población y muestra objeto de estudio han sido la totalidad de los (80) jugadores que componen los dos equipos mejor posicionados (A y B) de la cantera del club en la categoría cadete (15-16 años) masculina del club de fútbol Sevilla F.C.

\section{Diseño de estudio e instrumento}

Se llevó a cabo un estudio descriptivo y cuantitativo, implementando la herramienta MCJF de Lázaro et al. (2019), formada por 4 dimensiones (psicosocial, táctica, técnica y física), conformado por 10 criterios que analizan 37 variables (Figura 1). El Alfa de Cronbach obtenido en la herramienta fue de 0.90. Para este estudio se ha obtenido una valoración de 0.81 . Además de la reco- 
gida de datos sociodemográficos (edad, categoría y equipo), también se analiza a lo largo de este estudio la posición de juego donde se establecen siete puestos; central, delantero centro, extremo, lateral, mediapunta, mediocentro defensivo, mediocentro organizador (estas posiciones fueron evaluadas en cada equipo cadete durante la realización de este trabajo).

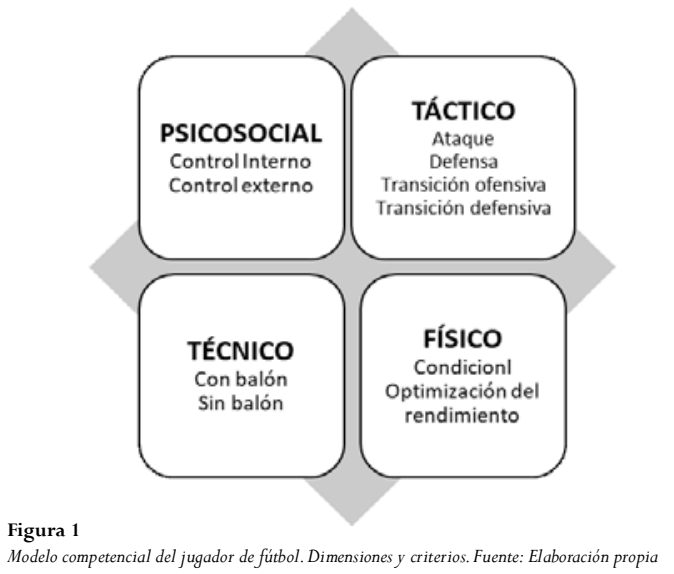

Las 37 variables permiten tanto la autoevaluación de la percepción del jugador como de la evaluación por parte del entrenador. Cada dimensión (cuatro) se ha evaluado conforme a una escala de Nivel competencial, de cero (carencia absoluta de la competencia) a Nivel competencial cinco (excelencia en la competencia evaluada) en un rango de valoración con una puntuación de cero a 100 (Tabla 1). Se describe una situación específica del desarrollo de la competencia y se evalúa en función del puesto que ocupa el jugador desde una doble perspectiva, la percepción por parte del entrenador y la autopercepción del propio jugador.

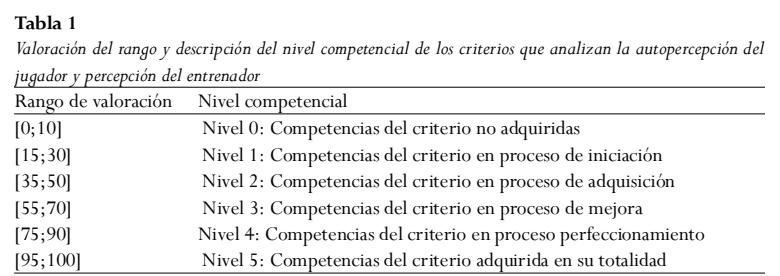

\section{Procedimiento}

Los entrenadores de los equipos de la categoría cadete (A y B), fueron contactados vía correo electrónico, informándoles de la finalidad del estudio. Se les informó de los objetivos e hipótesis de investigación y se les mostró el cuestionario y la propuesta del modelo MCJF. Posteriormente, en junio de 2018 fue realizada una jornada presencial de formación y capacitación de los cuerpos técnicos para dar las instrucciones con el fin de cumplimentar el cuestionario en papel, aclarando dudas y unificando criterios de evaluación. El plazo de entrega de los cuestionarios fue de una semana. Cada cuerpo técnico cumplimentó la evaluación de cada uno de sus jugadores de forma online. Previamente el club informó del estudio y solicitó el consentimiento a los tutores legales de los menores.

De igual forma se les explicó a los jugadores la metodología para que pudieran cumplimentar también el cuestionario. Cabe destacar que se informó reiteradas veces que el cuestionario fuera contestado de la forma más clara y con el máximo criterio posible, ya que el valor y utilidad del estudio queda condicionado por la veracidad de sus respuestas.

\section{Análisis de datos}

Se analizan las puntuaciones globales de las dimensiones y los criterios del modelo. En el estudio se ha realizado un análisis descriptivo para las dimensiones física, psicológica, técnica y táctica determinando la media y la desviación típica en función de la autopercepción de los jugadores y de la percepción de los entrenadores.

Fue realizado un análisis de varianza, ANOVA (de dos direcciones) atendiendo a la categoría cadete (A-B), desde la perspectiva de la autopercepción del jugador y de la percepción del entrenador para analizar la influencia sobre la posición que ocupa un jugador en el terreno de juego y sobre la valoración de las dimensiones psicológicas, tácticas, técnicas y física y la percepción que tiene su entrenador sobre estas competencias.

En el análisis de los datos fue utilizado el programa estadístico SPSS 21, (Statistical Package for the Social Sciences) con licencia de la Universitat Politècnica de València. Las pruebas estadísticas fueron realizadas con un nivel de confianza del 95\% (correspondiente a un nivel de significación de $p=.05)$.

\section{Resultados}

La finalidad de este estudio fue implementar una metodología de evaluación para el análisis de un modelo competencial del jugador de fútbol (MCJF) que permitiera posicionar a los jugadores en el nivel competencial correspondiente, y a su vez, aplicar el MCJF para poder analizar la autopercepción del jugador de fútbol y la percepción del entrenador sobre las valoraciones de las dimensiones competenciales en función del puesto que ocupa el jugador en el terreno de juego.

Análisis descriptivo de las dimensiones física, psicológica, técnica y táctica del modelo 
competencial y del rango de puntuación de los criterios respecto a los jugadores, entrenadores y categoría $(A-B)$

En la Tabla 2 se observa que entre las dimensiones (táctica, técnica, física y psicológica) de autopercepción de los jugadores con respecto a las puntuaciones obtenidas se sitúa en 68.8 en cadete A y 66 en cadete B. Entre ambas categorías, los cadetes $\mathrm{B}$ presentaron un porcentaje final más bajo en comparación con los cadetes A. En los jugadores cadete A la dimensión física presenta la valoración más alta (69.5) respecto a las demás dimensiones, mientras que en los jugadores cadete $\mathrm{B}$ la valoración más alta se obtuvo en la dimensión táctica (68.05). En cuanto al rango de puntuaciones comparando cada dimensión de los jugadores, se encontraron en el nivel tres de competencia, ya que la valoración en este nivel tres oscila entre los 55-70 puntos (Tabla 1). Esta puntuación supone que los jugadores se encuentran en un proceso de mejora, es decir, no hay una dimensión que destaque con respecto a otra.

En cuanto a la valoración que realiza el entrenador respecto al jugador en relación con las dimensiones tácticas, técnicas, físicas y psicológicas, el porcentaje entre las dimensiones se sitúa en 62.3 en cadete A y 59.6 en cadete $\mathrm{B}$, es decir, de la misma manera que los jugadores, el entrenador los categorizó en el nivel tres de competencia, ya que valores se encuentran en el rango de valoración 55-70 correspondiente al nivel tres (Tabla 1). Como se observa, la categoría cadete $B$ presenta una valoración entre las dimensiones (valoración final) menor en comparación con el grupo A (Tabla 2).

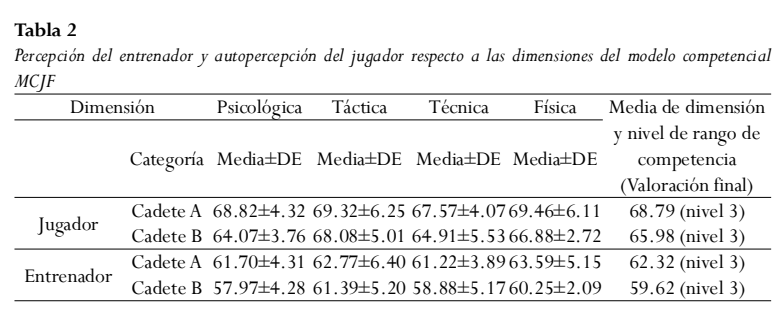

En la comparativa entre las categorías A y B, respecto a la autopercepción de los jugadores, mostró una tendencia similar en todas las competencias evaluadas, sin embargo, el grupo A presentó mayores porcentajes de puntuaciones en cada una de las dimensiones (Tabla 2). En las dimensiones psicológica (68.8) y física (69.5) es donde mayor diferencia hay entre estas categorías favoreciendo a la categoría A. Por otra parte, en la dimensión táctica, los porcentajes en las valoraciones presentaron pocas variaciones, encontrándose por porcentajes entre 69.3 (cadete A) y 68.1 (cadete B) (Tabla 2). De igual manera, en las percepciones de los entrenado- res respecto a los jugadores, las mayores diferencias entre las categorías A y B se observaron en las dimensiones psicológica y física (61.7 vs 52; 63.6 vs 60.3) (Tabla 2).

Análisis de la comparativa de los participantes respecto al modelo competencial y puesto ocupado en el campo de juego

En la Tabla 3 se aprecian los resultados obtenidos en cuanto a la diferencia entre la valoración que hace el entrenador y autopercepción del jugador, de cada una de las dimensiones que forman parte del modelo competencial en función de la posición que ocupa el jugador en el campo de juego. Es decir, en la tabla 3 se recogen los valores medios que corresponden a la resta entre la valoración que realiza el entrenador menos la valoración que realiza el jugador.

En la dimensión psicológica se muestra valores similares entre las valoraciones en las posiciones laterales y extremo. Los valores muestran que la diferencia entre la percepción del entrenador y de los jugadores es de 4.8 en jugadores con la posición de campo lateral y 4.6 en la posición de extremo (Tabla 3), esto indica una mayor concordancia entre percepciones de jugadores y entrenador en estas posiciones. Las mayores discrepancias se observaron en los mediocentro defensivo y mediocentro organizador, ya que se obtuvieron como valores 8.2 y 8.4 respectivamente. Por otro lado, en la dimensión táctica, a diferencia del ámbito psicológico, las mayores diferencias en percepción se observaron en los extremos y laterales (6.7) (Tabla 3).

En cuanto a la dimensión técnica la mayor diferencia se produce en la posición de mediapunta (7.1). En los puestos de mediocentros defensivos se observa una correspondencia similar en la valoración de esta dimensión (5.4).

En la dimensión física, se observan mayores diferencias en las posiciones extremo y de mediocentro organizador (6.9 y 6.6 respectivamente), mientras que la muestra estudiada mostró una mayor concordancia en la percepción con respecto a los laterales, ya que se

\begin{tabular}{|c|c|c|c|c|c|}
\hline \multirow{2}{*}{ Posiciones } & \multicolumn{4}{|c|}{ Dimensiones } & \multirow{2}{*}{$\begin{array}{c}\text { Media de los } \\
\text { jugadores (valoración } \\
\text { final) }\end{array}$} \\
\hline & Media \pm DE $(*)$ & $\frac{\text { lactica }}{\text { Media } \pm \mathrm{DE}(*}$ & Media $\pm \mathrm{DE}(*)$ & $\overline{M e d i a \pm D E}(*)$ & \\
\hline Central & $7.4 \pm 4.73$ & $6.02 \pm 3.72$ & $5.73 \pm 5.04$ & $6.21 \pm 4.12$ & 6.34 \\
\hline Delantero centro & $6.15 \pm 5.91$ & $5.79 \pm 4.20$ & $5.66 \pm 4.12$ & $6.29 \pm 3.53$ & 5.96 \\
\hline Extremo & $4.63 \pm 2.79$ & $6.72 \pm 4.54$ & $6.30 \pm 4.59$ & $6.89 \pm 5.10$ & 6.13 \\
\hline Lateral & $4.79 \pm 4.34$ & $6.72 \pm 5.80$ & $6.66 \pm 4.24$ & $5.88 \pm 3.34$ & 6.01 \\
\hline Mediapunta & $5.44 \pm 3.83$ & $6.54 \pm 4.79$ & $7.09 \pm 5.48$ & $6.50 \pm 5.89$ & 6.39 \\
\hline $\begin{array}{c}\text { Mediocentro } \\
\text { defensivo }\end{array}$ & $8.18 \pm 6.89$ & $6.44 \pm 4.29$ & $5.36 \pm 3,53$ & $6.62 \pm 3,53$ & 6.65 \\
\hline $\begin{array}{l}\text { Mediocentro } \\
\text { organizador }\end{array}$ & $8.42 \pm 6.75$ & $6.27 \pm 5.04$ & $6.0 \pm 4,41$ & $6.94 \pm 3,53$ & 6.91 \\
\hline
\end{tabular}


obtuvo un valor menor comparado al resto de las posiciones (5.9) (Tabla 3).

En la prueba estadística ANOVA se tuvo como factores, la posición del jugador con 7 puestos en el campo (central, delantero centro, extremo, lateral, mediapunta, mediocentro defensivo, mediocentro organizador). Las variables respuestas o dependientes vienen dadas por las valoraciones obtenidas en las dimensiones evaluadas por los jugadores (autopercepción) y la percepción del entrenador (Tabla 4).

En cuanto a la autopercepción de los jugadores respecto a las posiciones que puedan tener los jugadores en el campo, puede observarse que depende de la posición del jugador las dimensiones táctica y técnica, con valores de $p<.05$. De igual forma ocurre con respecto a la percepción del entrenador, se obtuvo valores de $\mathrm{p}$ inferiores al nivel de significancia en las dimensiones tácticos y técnicos. Para ambas muestras analizadas (jugador y entrenador) las dimensiones psicológica y física no mostraron valores de $\mathrm{p}$ significativos $\left(p^{>} .05\right)$.

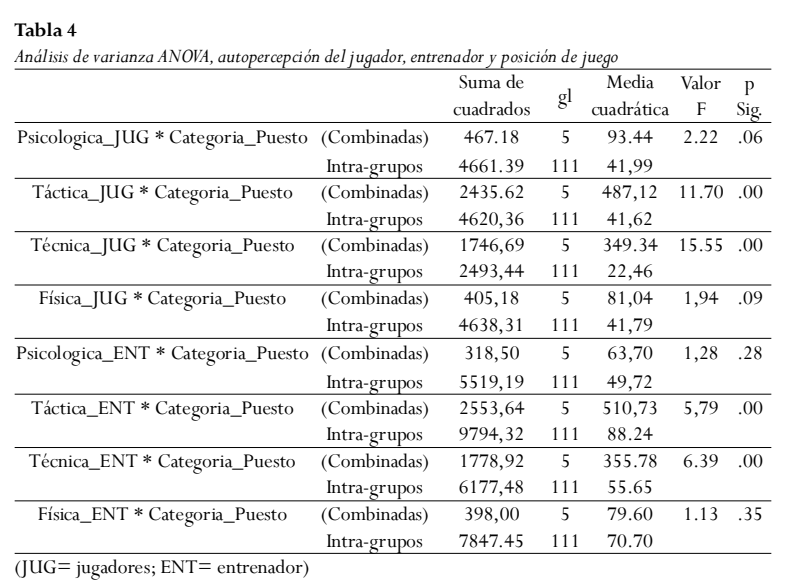

\section{Discusión}

Considerando que el objetivo del estudio ha sido implementar una metodología de evaluación para el análisis de un modelo competencial del jugador de fútbol (MCJF), atendiendo a la hipótesis 1 relativa a la existencia de diferencias entre las percepciones del jugador y del entrenador en las diferentes dimensiones (psicológica, física, técnica y táctica, esta se confirma. En todas las dimensiones el entrenador siempre valoraba más bajo respecto a la valoración de los jugadores; donde éstos en ocasiones mostraron una evaluación mucho más positiva. Por otra parte, se obtuvieron similitudes respecto a los equipos de cadete (A y B), ya que tanto los jugadores como sus entrenadores coinciden en que se encuentran en un nivel competencial en proceso de mejora (nivel 3).
Investigadores como Lago (2008) señalan que las diferencias observadas en los resultados de los partidos de fútbol son debidas, en mayor parte, a las técnicas de posesión de balón, planteamiento táctico en el juego y la defensa, es decir, estas características pueden establecer diferencias importantes entre equipos. Por su parte, Cunha et al. (2010) señalan que a medida que aumenta la experiencia en el balompié los jugadores aprenden a percibir adecuadamente cada factor que envuelve al fútbol, aumentan sus capacidades de juicio y reconocen sus límites como jugador, por ende, los jugadores juveniles (jugadores de mayor edad) pueden tener una mayor capacidad de autoanalizar, ofreciendo una impresión centrada en la realidad o una percepción con mayor similitud a la realizada por el entrenador.

Vega-Orozco et al. (2020) señalan que existe una relación positiva entre el nivel de pensamiento táctico y técnico con el desarrollo de estas habilidades, es decir, mientras mayor sean estos niveles, mayores serán los cambios que se producirán en sus habilidades, siendo estos cambios significativos. De ello se puede inferir que la diferencia en la experiencia profesional, la formación deportiva y mentalidad entre las edades de los jugadores puede dar lugar a opiniones y capacidades de autoanálisis muy distintas sobre los aspectos evaluados en el instrumento (Bettega et al., 2018).

Dichos resultados podrían indicar que los deportistas evaluados aún no perfeccionan las técnicas y tácticas de juego, ya que se encuentran en proceso de aprendizaje. Williams y Hodges (2005) establecen que las habilidades tácticas y técnicas de un jugador se aprende y mejoran cuando el jugador es puesto en situaciones donde sea necesario la resolución de problemas. El instrumento de evaluación MCJF se basa en dicha afirmación, donde son evaluados una serie de situaciones que posicionan a los jugadores en diferentes niveles dependiendo de cómo el jugador se desenvuelve en ellas (Lázaro et al., 2019).

De igual forma, los resultados obtenidos pueden estar relacionados con el estrés, las exigencias fisiológicas representadas por las intensidades a las cuales se realizan las actividades durante un partido y las demandas que implica trabajar en un entorno de alto rendimiento (Fernandes et al., 2019). Estas demandas podrían incidir en el nivel competencial de los jugadores cadetes y su posicionamiento en el nivel intermedio (nivel tres) que, al no dominar y comprender todavía por la edad, aún no tienen perfeccionadas las técnicas y tácticas de juego, encontrándose los jugadores en un proceso de aprendizaje. De igual forma, elementos como el estrés puede influir en la toma de decisiones de los jugadores 
(Almonacid-Fierro et al., 2020) pudiendo disminuir el nivel competencial de los jugadores.

Considerando este aspecto, se hace evidente que este tipo de valoración puede aportar mejoras en el proceso de evaluación del rendimiento deportivo, ya que los jugadores fueron capaces de analizar de forma critica sus dimensiones competenciales. La evaluación por medio de la percepción de los jugadores y entrenador permitió suministrar información relevante sobre el desenvolviendo psicológico, físico, táctico y técnico del jugador, así como también brindó información acerca de la capacidad del jugador en resolver diversas situaciones de juego que puedan estar presentes en los entrenamientos y en los partidos de fútbol. Se demostró que la metodología empleada, incorporando el modelo competencial en las evaluaciones del entrenador, pudo contribuir a que éste comprenda mejor las necesidades y problemas que enfrentan sus jugadores.

De igual manera, la metodología utilizada permitió un mayor acercamiento a la situación requerida por el entrenador en cada uno de las dimensiones evaluadas, por lo tanto, las valoraciones dadas por las percepciones ayudan a los futbolistas a mejorar su nivel de competencia y rendimiento deportivo, siendo afirmada la efectividad del instrumento. Este hecho potencialmente puede mejorar el proceso de evaluación y aprendizaje de los deportistas.

Autores como Estrada \& Pérez (2011) y Guillén (2010) señalan que la autoevaluación de los jugadores puede aumentar el rendimiento deportiva, ya que favorece la motivación y el control del estrés, la concentración, la dinámica con el equipo y permite aumentar la eficiencia del jugador (Estrada \& Pérez, 2011). De forma general, la metodología empleada utilizando la percepción de los jugadores sobre su propia capacidad para llevar a cabo una tarea de manera exitosa puede generar un impacto significativo en su rendimiento deportivo.

De acuerdo con estos autores en este estudio se considera adecuada la utilización de esta metodología en la valoración de las dimensiones competenciales, para así disponer de una información más completa y acertada sobre el rendimiento deportivo de los jugadores de fútbol (Castellano, 2018). De forma general, en el deporte colectivo de equipos base una de las causas que genera una disminución en el rendimiento es la falta o baja comunicación con los entrenadores (Álvarez-Medina, 2016; Urbano et al., 2020). La valoración de las competencias utilizando la percepción por ambas partes permite una comunicación abierta y, a su vez, conocer el estado del jugador (Álvarez-Medina, 2016; Casais, 2008), lo cual mejora los procesos de evaluación en el fútbol. Casais (2008) señala que la implicación del jugador en su propia evaluación se hace necesario, ya que la información obtenida de ello y el conjunto «entrenador-jugador» serán claves para llevar a cabo una evaluación real y ajustada a cada jugador.

Prieto (2017) afirma la necesidad de ofrecer a los jugadores más jóvenes una formación en el deporte más ajustada a sus necesidades, ya que éstos muestran menores grados de madurez en la asimilación de los aspectos tácticos y técnicos. La utilización de una metodología de evaluación centrada en las percepciones posibilita la obtención de las necesidades requeridas por los jugadores, ya que entre ambas partes se pueden llegar a un consenso en cuanto a requerimientos para mejorar el rendimiento del jugador

En cuanto a la hipótesis dos, la cual señala la existencia de diferencia entre las percepciones del jugador y del entrenador en las diferentes dimensiones (psicológica, física técnica y táctica) respecto a la posición del jugador en el campo, se obtuvo que solo las dimensiones técnicas y tácticas dependen del puesto que ocupa. Estos resultados permiten inferir que las diferencias observadas entre los jugadores en estas dimensiones son el resultado del efecto de la variable independiente y no del azar. Por otra parte, se puede inferir que las dimensiones como la psicológica y física son propias de cada jugador, las cuales pueden variar ampliamente sin encontrarse relacionadas con la posición que ocupa un jugador en el terreno de juego.

En el estudio realizado por Prieto (2017) se utilizó un instrumento de evaluación del rendimiento y formación de jugadores de fútbol en las categorías sub-10 a sub-23 de un equipo profesional de España. Este autor optó por una metodología observacional con el fin de poder evaluar el rendimiento técnico y táctico de los jugadores. Se encontró que el desempeño técnico y táctico fue evolucionando entre los jugadores. Las habilidades tácticas fueron progresando desde la portería hasta los jugadores ofensivos, los cuales exhibieron los mayores grados de habilidad en los criterios evaluados. Terry (2008) reportó resultados similares en cuanto a la evolución de las habilidades deportivas. Estos autores señalan que las evaluaciones de los jugadores dependen en gran medida de sus habilidades técnicas y tácticas, existiendo una correlación entre estas dimensiones con la posición del jugador.

Cabe destacar que no se han encontrado estudios similares que determinarán el nivel de competencia y 
rendimiento deportivo en jugadores de fútbol (Ravindranathan et al., 2017), a excepción de las investigaciones de Prieto (2017) y Terry (2008) mencionadas anteriormente, por lo tanto, se considera que este estudio es una aportación de utilidad para futuras investigaciones.

Este instrumento, siguiendo la metodología empleada, podrían ser aplicado en diferentes fases de evaluación, siendo propuesta tres evaluaciones, una inicial, otra durante las pruebas de entrenamiento y una final, lo cual potenciaría la mejora del proceso de evaluación en el fútbol. Esto proporcionaría un análisis completo de los jugadores y, además, sería información útil tanto para los entrenadores como para los deportistas, ya que, en algunos casos serviría para mejorar la planificación y control didáctico de los entrenadores en los entrenamientos y como fue mencionado anteriormente, brindaría conocimiento sobre las mayores debilidades de los jugadores, lo cual permitiría mejorar el rendimiento deportivo.

En base a este razonamiento se considera que esta metodología puede ser empleada cuando se quiere evaluar a los jugadores y examinar sus rendimientos deportivos para ser fichados. De igual manera, puede ser utilizada para realizar la evaluación competencial del jugador de fútbol durante el desempeño de su actividad deportiva en los entrenamientos y examinar las habilidades y capacidades del jugador durante las concentraciones y partidos de fútbol.

Además de esto, este instrumento puede ser utilizado cuando se quiere detectar las fallas en el rendimiento y especificar en donde es requerido realizar una intervención. El jugador por medio del proceso de retroalimentación con el entrenador puede informar de cómo ha mejorado su rendimiento y el entrenador puede comparar estas percepciones con sus observaciones. Esto implica que una de las ventajas que ofrece este tipo de valoración es que al ser una herramienta que puede ser aplicada por el mismo futbolista, éste también puede autoanalizar cuales áreas tiene de mejorar. Todo esto conlleva a mejorar la evaluación de los deportistas dentro de esta disciplina altamente demandada.

Por último, es de señalar que las percepciones de los entrenadores sobre los jugadores más hábiles podrían generar efectos contrarios en cuanto a mejora del rendimiento deportivo del equipo en general, ya que al tener una mejor percepción en estos jugadores la cantidad de tiempo dentro de la cancha de estos jugadores podría aumentar para asegurar el resultado positivo en algún partido. Esta percepción puede interferir directa- mente con la oportunidad de participación y desarrollo deportivo de otros jugadores, siendo así conceptualizados los efectos Pigmalión y Galatea dentro de este tipo de metodología. Es por ello que es de importancia considerar dichos efectos al momento de aplicar esta herramienta de evaluación.

\section{Conclusiones}

El análisis realizado evidencia que la autopercepción de las dimensiones psicológicas, tácticas, técnicas y físicas de los jugadores difieren de la percibida por los entrenadores, mostrando los jugadores en todos los casos, una valoración superior respecto a los entrenadores. Sin embargo, tanto para los jugadores como los entrenadores, hay coincidencia en la valoración del rango competencial, nivel tres, para ambas categorías A y B. Además, también se han obtenido resultados valiosos respecto a la posición del jugador en el campo, ya que las evaluaciones con respecto a las dimensiones tácticas y técnicas depende de la posición del jugador.

Una limitación a considerar es que se ha analizado una muestra participante de un mismo equipo, por lo que sería adecuado para futuros estudios analizar otros equipos de fútbol, además de la necesidad de aumentar la muestra de análisis.

Esta investigación evidencia unos resultados positivos empleando un instrumento válido y adecuado (Lázaro et al., 2009) para ayudar a los técnicos y entrenadores en los programas de preparación y evaluación de los futbolistas en categoría cadete como parte del procedimiento para detectar y analizar el talento de los jugadores en los diferentes niveles de los deportistas en las dimensiones analizadas de técnica, táctica, física y psicológica.

Concluyendo, la utilización de esta metodología conlleva unas implicaciones prácticas directas, por un lado, porque se trata de la primera herramienta que analiza las cuatro dimensiones (psicológica, técnica, táctica y física) de forma conjunta, y por otro, porque ello ayudará a identificar los puntos fuertes y las áreas de mejora de los jugadores cadete, para así adaptar las competencias del jugador al modelo de juego en equipo.

\section{Agradecimientos}

Los autores agradecen al Sevilla Fútbol Club el permitir realizar este estudio con los jugadores de sus equipos pertenecientes a la categoría cadete y por facilitar las áreas de trabajo. 


\section{Referencias}

Abad, M.T., Giménez, F. J., Robles, J., \& Castillo, E. (2013). La formación de los entrenadores de jóvenes futbolistas. Ebalonmano.com: Revista de Ciencias del Deporte, 9(2), 105 114.

Adanis, C. D. , \& Segura, C. D. (2017). Autoeficacia, ansiedad precompetitiva y percepción del rendimiento en jugadores de fútbol categorías sub 17 y juvenil. (tesis doctoral) Universidad Nacional, Heredia, Costa Rica.

Almonacid-Fierro,A., Martinez, M., \& Almonacid, M. (2020). Elementos que influyen en el proceso de toma de decisiones en deportes individuales de alto rendimiento: un estudio cualitativo (Elements that influence the process of decision-making in high-performance individual sports: a qualitative study). Retos, 38,341-348. https: / / doi.org/ 10.47197/retos.v38i38.73966

Álvarez-Medina, J., Murillo-Lorente, V., Usan-Supervía, P., Ros-Mar, R., \& Manonelles-Marqueta, P. (2016). Percepción subjetiva como método de control de la intensidad en fútbol sala. Retos, 30,9-14. https://doi.org/ 10.47197/retos.v0i30.35128

Ballester, I., Fernández, R., \& Parra-Camacho, D. (2020).Adaptación y validación de una escala para la evaluación del desempeño profesional del entrenador de fútbol en base a su formación permanente, nivel deTIC y autoevaluación (Adaptation and validation of a scale for the evaluation of the professional perf. Retos, 40,272-280. https: / / doi.org/10.47197/retos.v1i40.83157

Barreto, A., Abad, M., \& Giménez, F. (2020). Design and validation of a questionnaire to study the process of training football players. Revista de Ciencias del Deporte 16(2), 127 38.

Bettega, O., Scaglia,A., Nascimento, J., Ibáñez, S., \& Galatti, L. (2018). O ensino da tática e da técnica no futebol: concepção de treinadores das categorias de base. Retos. Nuevas tendencias en Educación Física, Deporte y Recreación, 33,112-117. doi: 10.47197/retos.v0i33.55503

Caicedo-Parada, S., \& Calderón-Vargas, M. (2020). Diseño y validación de un instrumento observacional para la valoración de acciones tácticas ofensivas en fútbol vatof. Retos, 38,306-311. https://doi.org/10.47197/ retos.v38i38.76622

Cano, O. (2001). Fútbol:entrenamiento global basado en la interpretación del juego. Sevilla:Wanceulen Editorial Deportiva.

Carmichael, F., Thomas, D., \&Ward, R. (2001). Production and Efficiency in Association Football. Journal of Sports Economics, 2(3),228-243. doi: 10.1177/ 152700250100200303

Casamichana, D. , \& Castellano, J. (2009). Análisis de los diferentes espacios individuales de interacción y los efectos en las conductas motrices de los jugadores: Aplicaciones al entrenamiento de fútbol. Motricidad: European Journal of Human Movement, 23,143-167.

Casais, L. (2008). Revisión de las estrategias para la prevención de lesiones en el deporte desde la actividad física. Apunts 157,30-40.

Castellano, J. (2018). Relación entre indicadores de rendimiento y el éxito en el fútbol profesional. Revista iberoamericana de psicología del ejercicio y el deporte, 13(1),4149.

Castillo, R. (2014). El efecto Pigmaliòn. ¿Hasta qué punto determina nuestro futuro la visión que los demás tienen de nosotros? (Tesis doctoral). Universidad pontificia de las comillas Madrid.

Cunhan, G., Mesquita, I., Moreno, M., Boleto,A., Tavares,T., \& Silva, P. (2010). Autopercepción de las competencias profesionales de los entrenadores de fútbol en función de la experiencia personal y de la formación académica. Cuadernos de Psicología del Deporte, 10(1),23-36. Recuperado 20 septiembre 2019 de https: / / revistas.um.es/cpd/ article/view/110261

De Mata, F. (1999). Propuesta metodológica de la preparación física del jugador de fútbol a partir de indicadores de motricidad en la competición. Tesis doctoral. Universidad Politécnica. Madrid, España. Recuperado 19 marzo 2019 dehttp: / / www.cafyd.com / REVISTA / ojs / index.php / bbddcafyd/article/view/120/93

Devís-Devís, J., Valenciano, J., Villamón, M., \& PérezSamaniego,V. (2010). Disciplinas y temas de estudio en las ciencias de la actividad física y el deporte. Revista Internacional de Medicina y Ciencias de la Actividad Física y el Deporte, 10(37), 150-66. Recuperado 20 septiembre 2019 de http: / / cdeporte.rediris.es / revista/revista37/ artdisciplinas147.htm

Estrada, O., \& Pérez, E. (2011). Edad, concentración y su influencia en el autocontrol de la ansiedad en deportista. Cuadernos de Psicología del Deporte, 11(2),89-96. Recuperado 01 septiembre 2019 de https: / / revistas.um.es/ cpd/article/view/133871

Fédération Internationale de Football Association, FIFA (2009). Copa mundial de la FIFA. Recuperado 20 agosto 2020 de https:/ /www.fifa.com/

Fernandes,T., Camerino, O., Garganta, J., Pereira, R., \& Barreira, D. (2019). Design and validation of an observational instrument for defence in soccer based on the dynamical systems theory. International Journal of Sports Science \& Coaching, 14(2),138-152. doi: 10.1177/ 1747954119827283.

Fernández-Rio, J., \& Méndez-Giménez, A. (2014). Talent detection and development in soccer: a review. Journal of Sport and Health Research, 6(1), 7-18. Recuperado 20 agosto 2020 de http://www.journalshr.com/index.php/ 
issues / 2014/52-vol-6-n1 -january-april-2014/187fernandez-rio-j-mendez-gimenez-a-2014-talentdetection-and-development-in-soccer-a-review-journalof-sport-and-health-research-617-18

García-Mas, A., Palou, P., Smith, R. E., Ponseti, X., Almeida, P., Lameiras J. \& Leiva, A. (2011). Ansiedad competitiva y clima motivacional en jóvenes futbolistas de competición, en relación con las habilidades y el rendimiento percibido por sus entrenadores. Revista de Psicología del Deporte, 20(1),197-207. Recuperado 14 agosto 2020 de https: / / archives.rpd-online.com/article/view/754.html

González-Campos, G., Campos-Mesa, M. C., \& RomeroGranados, S. (2015). Análisis de la influencia de la evaluación del rendimiento en jugadores de un equipo de fútbol. Retos, 25,85-89. https://doi.org/10.47197/ retos.v0i25.34486

González-Ponce, I., Leo, F., Jiménez, R., Sánchez-Oliva, D., Sarmento, H., Figueiredo,A., \& García-Calvo,T. (2018). Athletes' Perceptions of Coaching Competency andTeam Conflict in SportTeams: A Multilevel Analysis. European journal of sport science, 18(6),851-860. doi:10.1080/ 17461391.2018 .1461245

González-Víllora, S., García-López, L., Pastor-Vicedo, J., \& Contreras-Jordán, O. (2011). Tactical knowledge and decision making in young football players (10 years old). Journal of Sport Psychology, 20(1),79-97. Recuperado 20 agosto 2020 de https: / / www.redalyc.org/pdf/2351/ 235119302007.pdf

Guillén, N. (2010). Implicaciones de la Autoeficacia en el rendimiento deportivo. Pensamiento Psicológico, 3(9), 21 32. Recuperado 18 julio 2019 de https:// revistas.javerianacali.edu.co/index.php/ pensamientopsicologico/issue/view/8

Hancock, D. J., Adler, A. L., \& Cote, J. (2013). A proposed theoretical model to explain relative age effects in sport. European journal of sport science, 13(6),630-637. 10.1080/ 17461391.2013.775352

Holt, N. L., \& Mitchell,T. (2006). Talent development in English professional soccer. International Journal of Sport Psychology, 37(2/3),6-98. Recuperado de https:/ / www.cabdirect.org/cabdirect/abstract/20063233293

Krahenbühl,T., \& Leonardo, L. (2020). The relative age effect: coaches' choices as evidence of social influence on youth handball. Journal of Physical Education and Sport, 20(5),2460-67. doi:10.7752/jpes.2020.05337

Krogh, M. (2009).An Eye forTalent:Talent Identification and the «Practical Sense» of Top-Levell Soccer Coaches. Sociology of Sport Journal, 26,365-382. doi: 10.1123/ ssj.26.3.365

Lago, C. (2008). El análisis del rendimiento en el fútbol. Estado actual y perspectivas de futuro de la investigación. In J. Castellano (Ed.), Fútbol e innovación (pp. 89-103)
Wanceulen.

Lago-Ballesteros, J., Lago-Peñas, C., \& Rey, E. (2012). The effect of playing tactics and situational variables on achieving score-box possessions in a professional soccer team. Journal of Sports Sciences, 30(14),1455-1461. doi: 10.1080/02640414.2012.712715

Lázaro, C. B, Gallardo,A. M, Jabaloyes, J., \& Salazar, J. (2019). Diseño yValidación de un cuestionario para Entrenadores de Fútbol Acerca de las Competencias de Jugadores en Categorías Juvenil, Cadete e Infantil. Gymnasyum, 1, 1-19. Recuperado 08 enero 2020 de https: / / g-se.com/diseno-y-validacion-de-un-cuestionario-para-entrenadoresde-futbol-acerca-de-las-competencias-de-jugadores-encategorias-juvenil-cadete-e-infantil-2586-saw5d30ab47dc737

López-Ros, V., \& Sargatal, J. (2014). El aprendizaje de la acción táctica. Girona: Universitat de Girona, Servei de Publicacions: Càtedra d'Esporti Educació Física de la UdG. Martín-Barrero,A., Gutiérrez, I., \& Falces-Prieto, M. (2020). Análisis del modelo de juego en un equipo de fútbol profesional de la Bundesliga de Alemania. Estudio caso. Retos, 39,628-634. https://doi.org/10.47197/ retos.v0i39.79923

Martínez-Cabrera, F. I., Núñez-Sánchez, F. J., Muñoz-López, A., \& de Hoyo, M. (2020). Aceleraciones de alta intensidad en el fútbol. ¿Por qué es importante el método de evaluación?. Retos, 39,750-754. https://doi.org/ 10.47197/retos.v0i39.82281

Mendo,A., \& Morales, V. (2010). Psicología y fútbol profesional: caracterización de un reto pendiente. Apuntes de Psicología, 28(2),237-261. Recuperado 08 enero 2019 dehttp: / / www.apuntesdepsicologia.es/index.php/revista/article/view/215/217

Monteleone, M., \& Ortega, M. (Ed.) (2015). La construcción de un modelo de juego. Barcelona, España: Paidotribo.

Nuviala,A., Nuviala, R., Pérez-Ordás, R., Morán-Gámez, G., \& Falcón-Miguel, D. (2021). Incidencia del género y la edad sobre la calidad, satisfacción y valor percibido de los usuarios de actividades deportivas organizadas. Retos, 42,37-46. https://doi.org/10.47197/ retos.v42i0.83480

Pazo-Haro, C. I., Sáenz-López Buñuel, P., \& Fradua, L. (2012). Influencia del contexto deportivo en la formación de los futbolistas de la selección española de fútbol. Revista De Psicología Del Deporte, 21(2),291-299. Recuperado 08 enero 2019 de https: / / www.redalyc.org/pdf/2351/ 235126897009.pdf

Pazo-Haro, C. I., Sáenz-López, P., Fradua, L., Barata,A. J., \& Coehlo, M.J. (2011) La formación de los jugadores de fútbol de alta competición desde la perspectiva de los coordinadores de cantera. Apunts Educación Física y Deportes, 104(2), 56-65. doi: 10.5672/apunts.2014- 
0983.es.(2011/2). 104.06

Peña, G., Domínguez,A., \& González, M. (2017). Regulators of sports commitment from the perspective of practitioners. Review and adaptation of the degree of commitment to sport (CSQ) scale in different sports modalities. Journal of Sport and Health Research, 11(1),7990. Recuperado 11 junio 2019 de http:// www.journalshr.com/papers/Vol\%2011_N\%201/ JSHR\%20V11_1_7.pdf

Paixao, P., Giménez, F. J., \& Abad, M.T. (2019). Diseño y validación de un cuestionario para estudiar la formación de entrenadores de fútbol base. Retos. NuevasTendencias en Educación Física, Deporte y Recreación, 35(1),294-300. https: / /doi.org/10.47197/retos.v0i35.63262

Prieto-Andreu, J., \& Ramírez-Muñoz,A. (2020). Percepción de jugadores de fútbol jóvenes sobre el entorno deportivo. Retos, 39, 267-270. https://doi.org/ 10.47197/retos.v0i39.77014

Pulido, J., Sánchez-Oliva, D., Sánchez-Miguel, P., Leo, F. \& García-Calvo,T. (2016). Influencia de la formación de los entrenadores sobre la motivación de los deportistas. Revista Internacional de Medicina y Ciencias de la Actividad Física y el Deporte, 16(64),685-702. http://dx.doi.org/ 10.15366/rimcafd2016.64.005

Prieto,A. (2017). La formación del talento en el ámbito deportivo: la transferencia de los procesos formativos en el ámbito educativo al joven jugador de fútbol. Tesis doctoral. Universidad de Castilla-La Mancha: Castilla-La Mancha. Recuperado 11 junio 2019 de https://ruidera.uclm.es/xmlui/ bitstream/handle/10578/16640/ TESIS $\% 20$ Prieto $\% 20$ Ayuso.pdf?sequence $=1$ \&isAllowed $=\mathrm{y}$

Prieto, M. (2018). Detección deTalentos en Futbolistas a Través de Pruebas Físicas y Técnicas y la Opinión de los Entrenadores. Revista de educación física: renovar la teoría y la práctica, 150,11-17.

Ortega, E., Jiménez, J. M., Palao, J. M., \& Sainz, P. (2008). Diseño y validación de un cuestionario para valorar las preferencias y satisfacciones en jóvenes jugadoras de baloncesto. Cuadernos de Psicología del Deporte, 8(2), 39-58.

Ravindranathan, S., Ardakani, H. D., Pimental,A., Lee, J., Care, N., \& Clark, J.F. (2017). Performance monitoring of soccer players using physiological signals and predictive analytics. NSF I UCRC for Intelligent Maintenance Systems (IMS), 1 11. Recuperado 11 junio 2019 de https:// www.mfpt.org/wp-content/uploads/2019/10/Paper_-Ardakani.pdf

Reilly,T.,Williams,A. M., Nevill,A., \& Franks,A. (2000a).A multidisciplinary approach to talent identification in soccer. Journal of Sports Sciences, 18(19),695-702. doi: 10.1080/ 02640410050120078

Reilly,T., Bangsbo, J. \& \& Franks, A. (2000b).Anthropometric and physiological predispositions for elite soccer. Journal of Sports Sciences, 18,669-683. doi: 10.1080/ 02640410050120050

Santos, S., Mesquita, I., Graça, A., \& Rosado, A. (2010). Coaches' perceptions of competence and acknowledgement of training needs related to professional competences Journal of Sports Science and Medicine, 9(1), 62-70. Recuperado 18 julio 2019 de https:// www.ncbi.nlm.nih.gov/pmc/articles/PMC3737976/

Tapia-Flores,A., \& Hernández-Mendo,A. (2011). Una herramienta para estudiar la dirección de equipos en fútbol. Cuadernos de Psicología del Deporte, 11(2),105-113. Recuperado 18 julio 2019 de https: / / revistas.um.es/cpd/ article/view/133891/123261

Terry, C. (2008). Diseño de una herramienta observacional de evaluación técnico-táctica del fútbol para niños de 10-11 años (Cienfuegos, Cuba).Tesis doctoral. Universidad de Granada: Granada.

Urbano Arévalo, F., ManchaTriguero, D., Gómez Carmona, C., \& Gamonales Puerto, J. (2020). Influencia del perfil del entrenador en el diseño de tareas en fútbol-base. Estudio de casos (Influence of coach profile on the design of training tasks in initiation to football. A case study). Retos, 38,204-212. https: / / doi.org/10.47197/ retos.v38i38.74456

Vera-Haro, G., Pino Ortega, J., Romero Cerezo, C., \& Moreno Contreras, M. I. (2015). Propuesta de valoración técnico-táctica mediante una situación de Juego colectivo básico en el fútbol de iniciación. Retos, 12,29-35. https: / /doi.org/10.47197/retos.v0i12.35034.

Vega-Orozco, S., Bernal-Reyes, F., Gavotto-Nogales, O., Sarabia-Sainz, H., \& Horta Gim, M. (2020). Correlación entre pensamiento táctico y el desarrollo de las habilidades técnicas y físicas de jugadores de fútbol infantil. Retos, 41,138-142. https: / / doi.org/10.47197/ retos.v0i41.75647

Vidarte, J., Castiblanco, H., Villa, J., \& Ortega, A. (2019). Values of the resistance of the university football player in special conditions, of the city of Manizales (Colombia) (Valores de la resistencia del jugador de futbol universitario en condiciones especiales, de la ciudad de Manizales (Colombia)). Retos, 36,211-215. https://doi.org/ $10.47197 /$ retos.v36i36.66592

Williams, A. M., \& Hodges, N. J. (2005). Practice, instruction and skill acquisition in soccer: Challenging tradition. Journal of Sports Sciences, 23(6),637-650. doi: 10.1080/ 02640410400021328. 